\title{
QUI A FAIT ET DÉFAIT LES GOUVERNEMENTS. DEPUIS 1919
}

\author{
François Perin
}

CRISP | « Courrier hebdomadaire du CRISP »

1959/4 $\mathrm{n}^{\circ} 4 \mid$ pages 1 à 18

ISSN 0008-9664

Article disponible en ligne à l'adresse :

https://www.cairn.info/revue-courrier-hebdomadaire-du-crisp-1959-4-page-1.htm

Distribution électronique Cairn.info pour CRISP.

(C) CRISP. Tous droits réservés pour tous pays.

La reproduction ou représentation de cet article, notamment par photocopie, n'est autorisée que dans les limites des conditions générales d'utilisation du site ou, le cas échéant, des conditions générales de la licence souscrite par votre établissement. Toute autre reproduction ou représentation, en tout ou partie, sous quelque forme et de quelque manière que ce soit, est interdite sauf accord préalable et écrit de l'éditeur, en dehors des cas prévus par la législation en vigueur en France. Il est précisé que son stockage dans une base de données est également interdit. 


\section{CENTRE DE REGHERGHE ET D'INFORMATION SOCIO-POLITIQUES - G.R.I.S.P.}

16, RUE AUX LAINES, BRUXELLES 1

COURRIER HEBDOMADAIRE. $\frac{\mathbb{N}^{\circ} 4 .}{36 / . I 8(493) .}$

QUI A FAIT WT DEFAIT LES GOUVERNEMMENTS.

$($ I9I8 - I958)

In I958, la Belgique a connu trois gouvernements différents. Tous les départements ont eu au moins deux titujaires mais le Commerce Extérieur, les Colonies, I'Instruction Publique, I'Intérieur, la Justice, 1a Santé Publique et la Famille, en ont eu trois, Th I958, le département des Affaires Economiques a été géró par quatre ministres différents.

De I9I8 à I958, la Belgique a connu 23 crises gouvernementales de caractère politique (à l'exclusion de celles qui survionnont nomalement après les élections législatives).

Le phénomène des crises gouvernementales a dono sérieusement marqué le déroulement de la vie politique belge. Comment se forment les gouternoments ? quels sont les causes et les méconismes des crises ministérielles? Telles sont los deux questions étudiées ici par F. PERIN. L'étude couvre la période I9I8-I958.

SOMMAIRE:

I. IA FORMATION DU GOUVERNEMIENT.

Comment s'élabore la composition interne des gouvernements ? p. 5

II. CAUSES FH NECANISMIES DES CRISES MINISTERIELIESS.

1. Vote hostile du perlement

p. IT

2. Déseccord des ministres

3. Décision des états-majors des partis

p; 12

4. Pression d'un groupe parlementaire

p. I3

groupes parlementaires p. I4

III. RRILATIVITE DE L'INSTABIIITE MINISTTERIEHLE. 
QUI A FATT EI DEFAIT IES GOUVIIRNEWWENS. Depuis I9I9.

\section{La formation du gouvernement.}

$$
\text { 328.I6/.I8 ( } 493)
$$

Le Roi ne peut constituer un gouvernement sans que celui-ci n'obtienne, immédiatement après la nomination des ministres, la confiance des chambres par un vote sur une résolution expressément formulée.

Le Rol est donc tenu de former un gouvernement susceptible de recueillir la confiance des chambres. Son choix n'est pas personnel. Ia compositinn politique des chambres constitue en droit constitutionnel un élément légitimement déterminant ( I ).

Le Roi apprécie-t-il selil la situation politique? Evidemment non ; Ie monerque procède à des consultations devenues traditionnelles. Il consulte le président de la Chambre et celul du Sénat qui peuvent, on raison même de leur fonction, apprécier les chances d'une formule gouvernementale devant les assemblées dont ils dirigent les débats.

Le Roi consulte aussi le Premier Ministre démlssionnalre, les leaders des groupes parlementaires, notament ceux de l'opposition, les chefs de partis, les hommes les plus représentatifs de l'opinion.

La formation du gouvernement se fait en deux ou trois temps : Io Roi désigne parfois directenent le Premier Ministre, celui-cl compose onsuite la liste des ministres et la soumet au Roi. Parfols, avant de désigner le "formateur " du gouvernement, le RoI, dans les cas difficiles, désigne un "informatour " ( 2 ) qui se substitue à lui pour procéder à des consultations politiques et qui lui fait rapport sur la situation.

Comment le choix du Roi est-il finalement déterminé ? quelles sont les interventions décisivos?

Aucun parti ne réunissant la majorité absolue depuis I9I8 ( 3 ), 1a tâche du Roi est peu aisée; il ne lui suffit pas, comme en Angleterre, de se tourner vers le leader du parti le plus puissant.

Le fait de la période qui nous occupe est l'intervention directe des états-majors des partis politiques. On salt que ceux-cl se réunissent on congrès, organe souverain de chaque organisation politique, formé des délégués des diverses sections locales; ces congrès élisent des bureaux ou comltés centraux qui prennent une part active à la formation des gouvernements. Leur action est plus ou moins discrśte. Alucun parti n'a jamais été jusqu'à voter une résolution rendue publique pour déstgner formellement un candidat premier ministre.

TIT V. Ie chef de I'Etat et le gouvernement, H.J. Ganshof van der Meersch Revue de droit international et de droit comparé I950, p. I88.

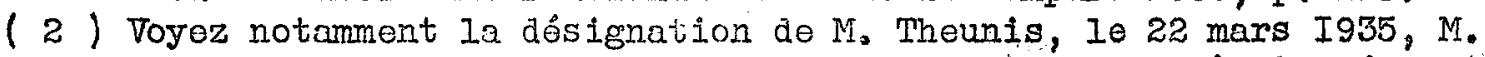
Tschoffen, lo ¿2 septembre I944; Mo de Schryver, oprès les élections du I7 février I946 et du ier juin I958.

(3) Sauf le parti social-chretien de I950 à I954. 
Les partis ont un minimum de respeot pour lo formalisme constitutionnel mais il est notoire que certains premiers ministres ont été délibérément choisis par leur propre parti ( I ).

La plupart du temps, Ie cholx de la personno du premier ministre résulte des négociations entaméos entre los personnalités politiques consultées par le chef de l'Etat.

Le premier ministre doit être non seulement agréé par son propre parti mais également par les partis qui foront partio de la coalition. C'est ainsi que $M$. Poullet a pu être présenté comme premier ministre après les élections du 5 arril I925 parce qu'il étalt la personnalité catholique qui manifestait le plus de compréhension et de sympathie à l'égerd du Parti ouvrier Belge qui allait entrer dans une coalition catholique-socialiste. II en est de même de M. P.H. Spaak, lors de la formation du gouvernement cathoIique-socialiste le 20 mars I947 et de M. Ejskens, cher de la coalition catholique-11bérale du II août I949.

Ia personne du premier ministre s'impose parfols par son attitude politique pendant la vie du gouvernement précédent. C'est alnsi qu'après $1 a$ chute du gouvernement Poullet-Vandervelde en I926, le Rol dásigna M. Jaspar come premier ministre; M. Jaspar était préc1sément le leader conservateur qui avait dirigé l'opposition contre le gouvernoment Poullet. Cette maniere de volr est mêne considérée comme un principe fondamental du régime parlementaire. Le Parti Ouvrier Belge fit au Roi un vif reproche de ne pas l'aroir appliqué après la crise du 27 février I924. Le gouvernement avalt été renversé à la Chambre par le vote conjugué des soclalistes et des catholiques flancnds

Lo Rol fot vivement attaqué pour n'avoir pas essayé de constituer un gouvernement à I'image de cette opposition ( 2 ).

Il arrive que des milieux distincts du monde politique jouent un rốle important dans la désignation du premier minlstre. Les milieux financlers et bancaires n'y sont pas étrangers ( 3 ).

Quelle est la part de l'influence personnelle du Roi ? Il est oxirmonant difficlia de répondre à cette question. I'action du monarque est couverte par une discrétion traditionnelle résultant du principe constitutionnel de 1 'irresponsabilité royale. Il est done impossible, faute de documents, de dégager avec précision le rôle personnel de la monarchie. Il parait certain que le Rol ne peut faire de cholx exolusivement personnel. Il ne peut donner libre cours à ses préférences politiques. Aucune désignation n'est le fait de la Cour ( 4 ).

( I ) C'est le cas notamment de M. Ach1lle Van Acker ( lo I2 février I945, Ie 3 I mars I946 et le 23 avril. I954) de M. Huysmans ( 3 août I946) ot de M. Van Houtte ( I5 janvier I952). M. Eyskens ( 4 juin I958).

( 2 ) Résolution du Conseil général du P.0.B. du 4 mars I924, A.P. Ch. , I9.3.I924, p. 673 et s. et 683 et s., Intervention de MM Hubin et Destréo.

( 3 ) Voyez notamment la désignation de M. Delacrolx le 22 novembre I9I8, de M. Theunis, le 5 décembre I92I et le I6 novembre I934, de M. Van Zeeland, Io 23 mars I935.

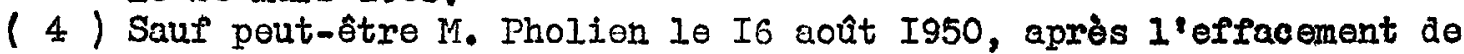
LéopolaIII, et encore, les réactions internes du P.S.C. n'y sont pas étrangères. 
Mals un Roi hablle, dans les moments difflciles, peut certes orlenter les ném goclations et faire surgir une solution qu"il appeIle personnellement de ses vooux. ( I )

Par exemple, il semble bien que le cholx de $M_{*}$ Carton de Wiart le 7 novembre I920 résulte vraisemblablement a'une ldée personnelle du Roi Albert, encore que celui-ci ait commencé par pressentir M. Seger's qui déclina l'offre de former le gouvernement. Il en est. de même de la personne de M. de Broqueville, premier ministre d octopre I932 à novembre I934; cet homme politique joulssait de toute la sympathie du Roi Albert ( 2 ).

Par contre, il n'est pas impossible que le Rol écarte des personnalités qui n'ont pas sa confiance. Il n'est pas rare non plus que le Roi manifeste publiquement ses préférences en pressentant une personnalité déterminée qui décline d'ailleurs cette invitation parce qu'elle ne correspond ni a ses vues ni à oelles de son parti. Ainsi, le I2 avril I939, le Roi Léopold offrit à Maspar le soin de former le gouvernement et au mois d'avril I954, le Ro1 Baudouin fit à M. Max Buset la même offre. Cette offre ne peut être interprétée comme un geste purement protocolaire.

Cette influence actire ne siexerce qu'd l'égard du premier ministre. Une fois, le formateur pressenti, $C^{7} 0 e^{4}$ lui qui dresse la liste de ses coéquiplers. Cette Iiste doit être soumise au Roi qui ne peut plus intervenir que sous forme d'exclusives discrètes, celles-ci n'aboutissant d'ailleurs à écarter un candidat que dans la mesure où le formateur $n^{\prime}$ a pas tenu lui-même à le consacrer.

Notons, toutefois ${ }_{2}$ qu'il est arrivé au Rot. de persuader personnelLement un candidat hésitant dentrer dans un ministêre ( 3 ).

Pour la composition du gouvernement; y compris le choix des personnes, l'intervention des partis est fréquente $\theta^{t}$ déterminante. File n'est pas exclusive, d'autres irfluerces se font souvent sentir.

Alnsi, le promior gouvernement formé par le Roi Albert après l'armistice du II novembre IOI3, présičé par M. Delacroix ( le 22 novembre I9I8), trouve son origine dans les négoeiations poursuivies au château de Lophem entre lo Roi et certains homes politiques. Le comité national de secours ot d'alimentation, dirigé par je financier Francqui, exerça une forte influm onoe sur la formation du gouverrement (4). Ce comité de pur fait avait aoquis pendant la guerre un prestige considérable en pays occupé tant par son action que par le personnalite de ses membres. $M_{0}$ Delacroix en faicalt d'aillours partie.

I'intervention das partis sure le programmu somme sur Ie choix des personnes fut évidento ( 5 )

( I ) Voyez I'attitude du kol Aiberí entre le 27 fétrier et lo 6 mars I924 lors du choix du premier ministre Theunis ( Höjer op o cito, po I40).

(2) Höjer, op. cit, p, 208,

( 3 ) ex. : M, Hubert dans 10 gouvernement Theunis: $\nabla$. la presse des I3 et I4

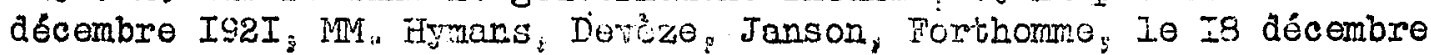
I932. Hbjers op cit。 $\mathrm{p}_{0} 208$ - 2I:

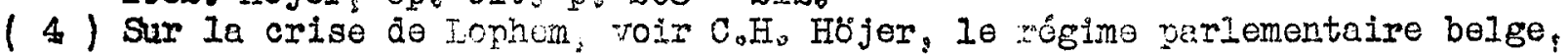
p. 63 à 90. Sur le Conseil national, idem, $p_{c} 70$.

(5) V. résolution des gnuches libérales du I6 ootobre I9Ig, rósolution du conseil général du P. O. B。 du 50 octobre I9I8 et du Is novembre - Höjers p. 79 et $8 \%$ 。 
5.

Les futurs ministres socialistos furent nantis d"uneautorisation de leur parti exprimée par un vote du conseil général. ( I ).

Le deuxième gouvernement présidé par ${ }^{\pi}$. Delacrolx fut influenoé de la même marière par les partis. ( 2 ).

Les gouvernementa Pormés par $\mathrm{M}_{\varepsilon}$ Carton de wiart le 23 novembre I920 ( 3 ), MM. Poullet-Vancierve:de Ie 23 juln I925, Jaspar Ie 20 mai I926 ( 3 ), Renkin ie 5 juli I93I, De Broquevilie le 22 octobre I932, et 10 I7 décembre I932 Van Zeeland le I2 décembre I935: Plerlot le I7 avril I939 résultèrent de négociations parfois longues et difficiles entre personnalités politiques, plus ou moins formellement mandatées par leurs Eroupes polltiques ou certains organes de leurs partis.

L'intervention du Conseil national du parti. libéral d'une part, du Congrès, ou du Conselz général du $P_{\diamond}, O_{,} B_{*}$ d'autre part, est à peu près constante : du côté catholique, celie de la Fédération des cercles et de la Iigue nationale des trivailleur's chrétiens $n$ iest pas rare.

Après I945, l'intervention des partis est régulière mais elle a donné lieu à beaucoup moins de difficultés qu'entre I9I8 et I939.

La situation politique qui mit le plus de temps à se dénouer est celle qui rósulta dos ézections de févxier I946. En dehors de co cas, les gouvernements se constituèrent assez aisément. Le róle de la monarchio fut donc infiniment plus effacé qu'entre les deurs guerres.

I'intervention des milieux "nanciers, dont certaines personnalités sont directement consultés par le Roi ou par les homes politiques, est particulièrement marquante dans ia folmation des gouvernements présidés par MM. Delacroix ( 22 novembre I9IS), Theunis ( 10 I6 décenbre I92I), Jaspar ( Ie 20 mai I925), Theunis ( le 20 novembro I934) et Von Zeeland ( Ie 25 mai I935). Lors de la formation de ce dern la première fois, consulta égalementi les représentants des grandes organisam tions économiques etu syndicalos.

I'Intervention des milieux bancaires est moins ostensible après I945 mais il faut néanmotns falre observer que deux ministres des finances furent des banquiers. (i).

\section{$++$ \\ Comment giélabore la composition dnterne des gouvernements?}

Ie nombro de zlinistzos d "uze tenäance politique déterminée est-il proportionnel à Ia furce de cet te temdance au parlement ? In calcul proportionnel purement ari.thmétiquo ust impossibie; si une certaine proportion est respeotée en ce qui. corcure les catholiques et les socialistes; on peut

TIT $\bar{V}_{0}$ Hö jer $; p_{0} I O 2-I 03$.

(2) idem. $p_{0}$ II2.

(3) Lintervention di Roi fü particuliérement active au cours des négooia. tions. Le cabinet du Foi publia des commuqués le I5 et le 22 ma1 I926.

(4) $M_{0}$ Gutt et $A_{0} E_{\text {。 }}$ Janssen.

En outre, il faut citer les ministises De Voghel (Banque Nationale) ot Jo Vauthier ( Ifquidateur a'organismes financiers issus de la guerre, occupant actuallement uno fonction inportanto à la Banquo do Brumelles. 
constater que la proportion des miniatres libéraux est constamment surévaluéo par rapport à la force parlementaire de leur part1. Ce phénomène est dú, croyons-nous, à la position centrale de ce parti dont l'appul est constamment recherché par les éventuels partenaires gouvernementaux. On a reproché aux libéraux leur goût disproportionné pour les portefeuilles ministériels. Mais l'histoire parlementaire prouve qu'ils ont constamment sollicités par les autres partis ou par la couronne elle-même. I'absence de majorité absolue et la répugnance naturello que catholiques et socialistes ont eu a s'associer. ont fait des libéraux des partenaires indispensables. Les conservateurs catholiques s'inquiétent à I'ldée de laisser les libéraux dans l'opposition, leur tondance économique et sociale étant fort scuvent la même que la leur. Le parti socialiste dont la tondance radicale est manifeste, sturtout en halionie, répugne également, pour d'autres motifs, à laisser les libéraux on dehors des responsabilités ministérielles. Ils craignent que le sentiment anticlérical de leur clientèle électorale no soit exploité par le perti libéral, alors qu'ils sont llés per une alliance gouvernementale aveo le perti qu"1ls qualifient eux-mêmes péjorativement de clérical. Cecl explique peut-être la raison pour laquelle, sur 40 ans de régime de suffrage unlversel, los libéraux totalisent 32 ennées de participation ministérielle.

Le dosage des portefeuilies ministériels n'est pas seulement déterminé par l'appartenance à un partl politique. Le dualité ethnique du pays, les mouvements flemends et wallons ont fortement influence la composition de tous les gouvernements. Fnin, les classes sociales différentes exigent une représentation spéciejie ( I). Les agriculteurs, les classes moyennes, les milleux syndicaux, sont particulièrement susueptibles à cet égard.

La compétence personnelle joue parfois un rôle déterminant; la préférence de M. Hymans et M. P.H. Spaak pour les affaires étrangères est évidente. Celle de $M$. Léfebvre, ministre de l'agriculture on I946 et de I954 a I958, se justifie par uno activité essentlellement orientée vers l'agriculture $(2)$, I'inclination de M. Plerre Vermeylen pour le département de $I^{\prime}$ intérieur (I947- I949-I954 - I958) n'est pas non plus l'effet d'un hasard ( 3 ). La défense nationale a tu plusieurs offiolers supérleurs investis de la fonction de ministre. Nous avons signalé à plusieurs reprises la présence de banquiers ou de financiers à la tête du département des finances. Mais, par aillours rèrno I'Indítornination la pilus oorplète.

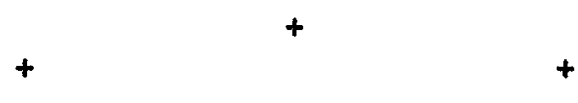

Ie principe même de l'intervention des partis a été exprimé formellement à maintes reprises.

C'est ainsi que le Congrès Ilbéral des I6-I8 octobre I920, dans sa résolution déclarait que "l'adhésion dét nitítve de mandataires libéraux à un cabinet en formation sera subordonnée à la ratification préalable par 10 congrès libéral des bases polltiques, économique et sociale sur lesquelles Ie ministerre entendra se constituer ".

(I) Surtout au sein du parti catholique.

(2) Il est lui-même propriétaire et dirigeant d'une importante exploitation agricole.

( 3 ) M. Vermeylon, arocat et professeur à I'U.L.B., dirige uno importante revue de droit communal i" "Encyclopédie dejurisprudence en matière d'affaires communales. Edition $U_{0} G_{\theta} A$.". 
D'autre pert, on peut lire dans"te Peuple " du 8 juin I925, Lors de la formation du gouvernement Poullet-Vandervelde, que "Io ConselI général s'est réunt ... pour discuter des c andidatures ministérielles ... Après un bref débat, le ohoix s'est porté sur les cltoyens Vandervelde, Wauters, Anseele, Huysmans et Laboulle ".

I'intervention des organes des partis dans la désignation des ministres n'est évidemment pas toujours aussi flagrante. II semble que, surtout après I945, si l'intervention des organes des partis, congrès, consells généraux, comités centraux, etc., est constatée on ce quil concerne les programmes et le princlpe même de la participation gouvernementale, les questionsde personne relèvent en fait de comftés plus restreints et plus secrets.

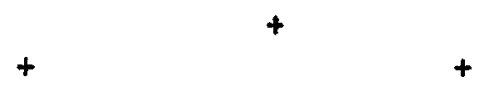

L'ingérence des partis dans la constitution des gouvernements $n^{i} a$ pas toujours été sans heurts ni sans réactions. Elle a soulevé des controverses passionnées surtout lorsque cette ingérence paralysalt la formation du cabinet. Ces réactions eurent une double origine: 1'opinion publique qui s'impatientalt de la carence des institutions politiques et le Roi mis devant des situetions extrêmement délicates que l'intervention excessive des partis transformait en impasses.

A titre d'exemple, soulignons le caractère laborieux des négociations qui présidèrent à la formation du gouvernement Van Zeelana au mois de juin I936 après. les éleotions du 24 mai. Ie formateur déclara à la presse qu'il ne rencontrait pas de difficultés insurmontables en ce qui concerne le programe mais que la répartition des portefeullies était tellenent ardue en raison des exclusires que les partis prononcèrent contre les personnes, qu'il devait finalement renoncer à sa mission. ( I ).

Pour faire pression sur les pertis, M. Van Zeeland, en pleine négoclation, s'adressa au public par la Radio lo 9 juin I936 en brossant un tableau des étónements et retraçant les grandes lignes de son programme, en rain d'allleurs, puisqu'il renonça quelques jours après è former le gouvernement. On proó́dé aussi inusité ne s'explique que par un sentiment d'irritation dans l'opinion publique, sentiment que le futur premier ministre entendait exploiter pour sortir de l'impasse. C'est finalement le Rol qui vint à son secours. II prit l'initiative devant son échec, de réunir les principales personnalités politiques en cause pour qu'elles se hâtent de mettre fin à la carenoe gouvernementale. Le Rol auralt fait à ses interlocuteurs de vifs reproches. "Il est incompréhensible " aurait-il dit " que des quéstions de dosages politiques et de personnes prolongent la carence gouvernementale, alors que les partis sont d'accord sur le programmo ( 2 ).

Le Rol aurait également fait pression sur les homes politiques responsables en les menaçant de dissoudre les chambres législatives, ce que 1a Constitution lui permet de faire avec un seul contreseing ministériel ( 3$)$.

Le résultat fut que $M$. Van Zeeland reprit les négociations, cette fois en dehors des partis et des groupes et constitua son gouvernement le jour même. Il n'est pas inutile de noter qu'une grève des mineurs avait été annoncée pour le I5 juin. Les élections avaient enregistré le succès relatif

$(I)$ V. Lo Solr, du IJ juin I936.
$\left(\begin{array}{l}2 \\ 3\end{array}\right)$ Ie Fopold III, Dumont, p. I74.
Io Flombeau, juillet I936, p. II4 et s. 
du part1 Rexiste ( I ). Une crise politique doublée d'une criso sociale ot économique se serait muée en crise du régime.

Lors de la formation du gouvernement Janson en novembre I937, 10 Roi, devant les difficultés suscitées por les négociations, fit une observation du même genre : "Il y a contradiction entre la volonté d'union nationale des partis et la corence à laquelle aboutissent les efforts des formateurs de gourernement. La situation actuelio ne peut se prolonger ( 2 ).

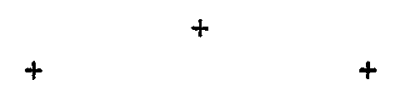

Les réactions royales et celles de l'opinion publique, la montée du fascisme en Burope exercòrent sur la formation des gouvernements une influence certalne. Le régime s'est réellement cru en danger ( 3 ).

Aiu mois de mai I938, après la démission du gouvernement Janson, M. P.H. Spaok, choisi personnellement par le Rol, constitus son équipe ministórlelle on molns de deux jours, sans consulter ni les partis ni les groupes parlementaires. Il fit connaftre la composition de son ministère lo I5 mal, la crise avait éclaté le I3. Fortement influencée par le Roi, la décleration mion. nistérielle contenait une allusion à une révision constitutionnelle tendant notamment à renforcer le pouroir exécutif, ce qui provoqua une réactlon énerglque de tous les partis. $(4)$.

Le premier ministre dénonça expressément l'habltude désagréable de falre intervenir les partis dans les négociations précédant la formation des gouvernements. Coux-ci doivent être indépendants des partis aussi bien que des groupes parlementaires. Le gouvernement doit avoir de l'autorité; lo parlenent ne doit exercer qu'un droit de contrôle. Les libéraux y virent un retour à le puroté constitutionnelle $(5)$, et les catholiques une heureuse tendance à renforcer l'autorité royale dont le gouvernement devalt essentiellement tirer la sienne $(6)$. Mais il ne fut question nulle part d'une réelle tentative de revision constitutionnelle.

En réalité, ce gouvernement " fort "qui no dura pas un an (7) no se distingua que pa la manière rapide dont 11 fut fo rmé. Le premier ministre ne put écarter $l$ ? ingérence des partis qu'on ce qul concerne lo cholx des personnes ( 8 ). Peu soutenu par son propre parti, M. Spaak subit plus fortement encore l'influence des partis $y$ compris le slen, précisément poux lo motif qu'on se pessant d'oux pour former son gouvernement, il leur conféra une indépendano qui le mit constamment en péril.

Cette faiblesse fit persister le Roi dans sa tendance au Lisou de I'en décourager. Le I2 février Iots, il chargea un de ses intimes, $M_{0} H_{0}$ Jaspar, retiré de la vie politique depuis quelques années et auquel les partis no songeaient plus, de la mission de former un gouvernement " fort " en dehors de toute Ingérence des partis, composé au moins pour moitié d'extra parlementaires. Il dut renoncer fmmédiatement à son projet. Le I4 férrier, le burequ

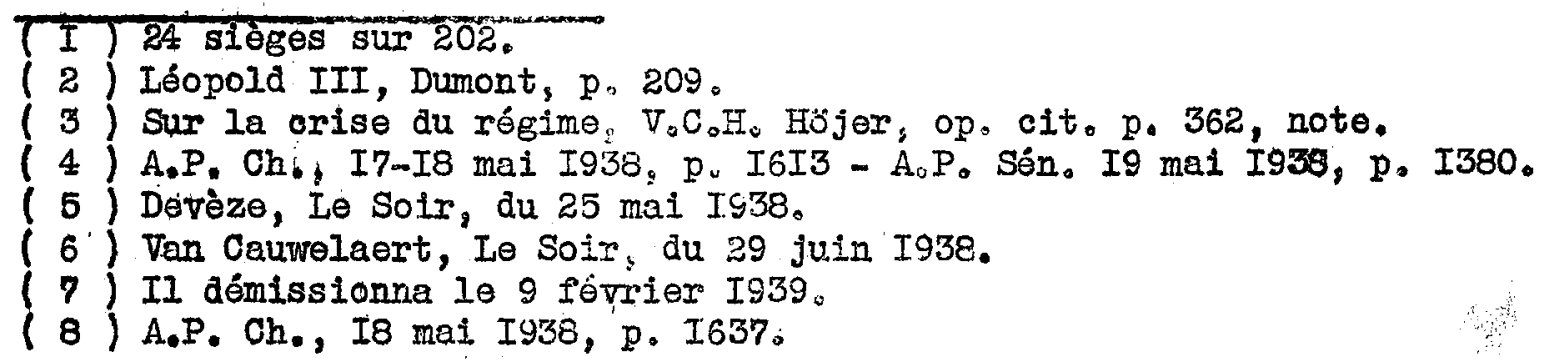


du P.O.B. publia un communiqué motivant le refus opposé à M. Jaspar. "Ie nombre anormal d'extra parlementaires ne se justiflait pas ". Ie minsitère constitué immédiatement après par M. Pierlot on comptait tout de même cinq sur onze ministres.

Le cabinet avait été constitué sans arrêter de progranme. M. Plerlot confla à la presse le $2 I$ février I939 qu'en outre 11 n'avait pas l'intention de demender un vote de oo nflance aux chambres. Celles-cl le jugeralent à ses actes. Le réaction du parlement fut telle que le gouvernement se soumit néanmolns à un vote mals par ce voto la chambre se contenta d'adopter un simplo ordre du jour prenant acte de la formation du gouvernement. Cotte tentative fut un éohec complet, le 27 du même mols, le gouvernement démissionna et un décret de dissolution des chambres fut signé le 6 mars. Les élections eurent lieu le 2 avrll I939. Une lettre du Rol au premler ministre du 6 mars I939 fut rendue publique. Le Roi evalt exposé ses principes au conseil des minlstres des 2 et 7 férrier et, lors de la réunion des principales personnalités politiques du moment, le IZ avril I939 au Palais Rogral ( I ).

"La première condition qui s'impose " disait le Rol," celle dont dépend, je n'hésite pas à l'affirmer, le sort même du régime, c'est la restauration dans toute son indépendance ot dans tout sa capacité d'action, d'un pourolr exécutif vraiment responsable, c'ost - à -dire formé d'hommes qui pulssent essurer le gouvernement du pays penaant toute une léglslature si possible, sens se trouver entravés dans leur action par des mots d'ordre de partis, par des décisions de groupes et de sous-groupes politiques ou par des préocoupations partisanes."

Ies efforts du souverain furent vains. Le P.O.B., par un vote de son congrès du I7 avril I939, refusa la participation au gouvornement. Les ministres socialistes démlsslonnèrent et les "ministrables " refusèrent les portefeuilles qu'on lour offrait. M. Pierlot forma une coalition catholiqueilbéralo. Cello-ci ne s'élargit aroo la participation des sociatistes que lo 3 septembre I939, sans I'Intervention des partis et des groupes mais dans le consentement général puisque la guerre venalt d'éclater à nos frontières. On remaniement out lieu le 5 janvier I940 sans consultation des groupes, $c \theta$ qui provoqua le protestation des partis. ( 2 ).

Le premier ministre évita la difficultéen refusant de répondre aux interpellations parlementaires à ce sujet $(3)$.

Clest dono surtout à partir de I936 que la tendance à secouer la tutelle des partis se manifosta avec un succès très mitigé.

II est néanmoins utile de signaler que les partis n'intervinrent pratiquement pas dans la formation de certains gourernements, antérieurs a cette arnée. Il en est alnsi du gouvernement Theunis, formé le I8 mars I924: du gouvernement Jaspar, le 23 novembre I927, du gouvernement.Renkin, du 23 mai I932, du gouvernement $\mathrm{De}$ Broqueville, le 8 juin I934. Mais, à vrai dire, ces gouvernements succédalent à d'autres cabinets prósidés par le même premier ministre. Ces homos qui avalent dirigé une équipe ministérielle déterminée et qui étalent chargés de la mission de former un nouveau ministère se comportalent essentlellement comme s'ils procédaient à un simple remaniement de leur gouvernement, dont la base polltique restait très souvent la

\footnotetext{
(I) LéopoldiII, Dumont, p. 227 et 237:

(2) Le 9 janvior I940. Comité directeur de la l1gue nationale des travail.leurs chrétiens.-Ie 7 janvier I940. Comité permanent du parti. libéral ( Le Soir du I7 janvier I940).

( 3 ) A.P. Ch. I7.I.I940, p. 324 - A.P. Son. I7.I.I940, p. 309 。
} 
même ( exeoption falte pour le gouvernement Jaspar du 23 novembre I927 qui perdait l'appul et la participation des sociallstes l.

Ia formation de ces gouvernements, mis sur pied avec l'accord taclte des pertis intéressés, n'a d'ailleurs pas provoqué au sein decceux-ci les vires réactions qui caractérisèrent les tentat1ves des années I936-I939.

I'instabilité du régime qui prit un aspect aigu pendant les deux années quil précédèrent immédiatement la guerre explique sans doute, en bonne partie, l'attitude du Roi Léopold III et a vraisemblablement contribué à creuser entre lul et les hommes politiques le fossé qui allait les séparer le $28 \mathrm{mal}$ I940. Le drame monarchique qui devait déchirer la Belg1que de I945 à I950 trouve peut-être sa source autant dans le mauvais fonctionnement du régtime à cette époque que dans les circonstances de la guerre.

La conclusion de cette analyse du mode de formation des gourernements se dégage arec netteté. Tout le régime est fondé sur l'exlstence de partis fortement organisés dont les états-majors négocient entre eux.

Le pouvoir réel est entre leurs mains. Cet état de fait a risqué à de multiples reprises, surtout entre I9I8 et I939, de provoquer des impasses.

Si les états-majors des partis n'aboutissent pas a une solution, le Rol Intervient avec énergie et relance les négociations qui risquent de s'enliser. I'absence de majorité absolue a donc donné ainsi à la monerchie un róle aotif assez paradoxal au XXe siècle. Mals ce róle n'a pu aller au-delà d'umeprésidence active de la vie politique. Toute vellélté de pouvolr personnel étalt vouée à l'échec, de même que toute tentative d'évincer réellement les partis de la formation des gouvernements. L'institution constitutionnelie la plus dépourvue de toute influence est le parlement lui-mêne. Ce dernier joue le róle de tribune politique et entérine par un vote, le résultat de négociations menées en grande partie en dehors de lui. Les groupes parlementaires ont joú sans doute un rôle incontestable, surtout à droite. Mais cos groupes apparaissent eux-mêmes comme les organes des machines polltiques que sont les partis. Cecl est surtout vrai pour les partis de gauche, mais le P.S.C. lui-même présente une structure fort proche de ses concurrents.

\section{CAUSSES EI MHCANISMIES DES CRISES MINISTERIETTLES.}

Hous detons exclure de l'analyse des crises ministérielles, les crises constitutionnelles : tout gouvernement doit être investi de la confianco des chambres législatives, il est dono normal qu'il s'en constitue un après chaque élection. Cette conséquence n'est d'alileurs pas absolue; au sein des chambres nouvellement élues, une majorité favorable au deraler gouvernement on fonotion peut lui falre confiance. Ce fut le cas lors des élections du 26 mal I929; le gouvernement de M. Iaspar, constitué en novembre I927 par une coalition du parti cathollque et du parti ilbéral, resta en fonction après aroir subl un très léger remoniement. Ie cas, est-1l besoin de le dire, est unique. Il faut éfolement exclure les démissions offertes au souverain lors de son accessief au trône. Elles font partif du cérémoniel et sont toujours refusées: elles n'ont d'allleurs aucune portée politique. Ia seule exoeption la règle est la formation du gouvernement de M. Pholien, en août I950, après l'accession du Prince Baudouin à la régence du royaume. Ia démission du gouvernement précédent, présidé par M. Duvieusart, fut acceptée pour des raisons pohitiques, Mais cette accession au trône est insolite dans l'histolre de la monarchie belge et Intervint après des événements révolutionnaires qui mirent l'existen$c \theta$ même de la manarchio et du pays en danger. 
Les causes que nous ênumérons lci sont des causes purement politiques, à l'exclusion des élections léglslatives. Elles interviennent en cours de législature.

\section{X}

De I9I8 à IS5s les gouvernements belges ont démissionné vingt-deux fols pour des motifs politiques. Sur vingt-deux crises gouvernementales, huit portent sur la politique économique, sociale, monétalre ou fiscale du gouvernement, quatre sur la po ique linguistique ouethnique, quatre sur la politique étrangère et militaire, deux sur la personne même diun ministre, une sur une réforme électorale, le suffrage féminin, enfin, deux sur la question royale pendant la grave crise monorchique que traversa la Belgique do I945 à I950.

Quel est le mécanisme cies crises gouvernementales?

Les dêmissions des gouvernements peuvent être classées en plusieurs catégories. Certains gouvernements sont renversés par un vote hostile d'une des deux chambres du parlement. $D^{i}$ autres se disloquent è cause du désaccord des ministres sur des questions politiquos importantes. Ia décision de mettre fin la vie d'un gouvernement peut être prise par les états majors des partis. Les ministres peuvert démissionner sous lo pression d'un groupe parlementaire. Ils peuvent aussi démissionner pour faire prassion sur un groupe porlementaire et un parti. Ils peuvent enfin êtro débordés par une crise économique et financière, et dámissionner spontanément sens que personne ne 10 Leur demande.

\section{Vote hostile du parlement:}

Depuls I9I8, deux gouveriements seulement furent renversés por lo parlement ( I ). Le gouvernement catholique-Iibéral de M. Theunis, le 27 février I924, et le gouvernement socialistemibóral-communisto de M. Van Acker, le 9 juillet I94ô. Le premjer succombait, devant une opposition hétérogèno socialiste et catholique flamando au sujet de la politique śtrangère. Les soolalistes étaient hostizes à l:0voupation de la Ruhr par les troupes belges, et $\nabla$ ingt catholiques flamands désiraient manifester leur hostilité à la po.. litique, trop francophile à Ieu: coût, du ministre des affaires étrangères Hymans. Le vote arait pour objet une convention commerciale avec la France, mois le débat siétait élargí jusqu"à embrasser toute la politique extérieure du gouvernement $i 2 \%$

Ce vote est caracténiś par sa confusion puisqu il a été inspiré par des mobiles divers très cirangcrs les uns aux autres: trois questions clalres en elles-mêmes ont été mêliées: la poiltique francophile du ministre, l'occupation de la Ruhr et la convention comeraiale. Cette crise fut parfaitement inutile puisque $M_{2}$ Theunis co:1stivia, Io Io mas I924, un gourernement fondé à peu près sur les mêmes kasos que ie précóćent.

Quont à la criso du juiliet Ióse

( 3 ), ello aboutits à la revonstitution d" somblable au précéderi maŝs arec un autio premier ministro (4).

TI le professour Mast en comptu cing. Nous 1 avors pas tenu compte des deux

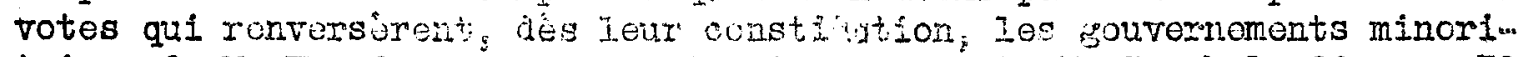

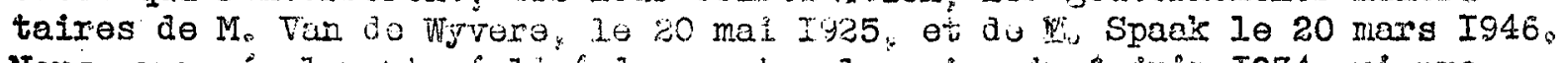
Nous avons également rógligé ce compter la oriso du 6 juin I934 qui prow voqua un sinple remaniement, du gouvernement de Broqueville of Jourial des

( 2 ) C.H. HöjeI : Op. cito, po.I3s

( 3 ) incident Roin warklabbalse。

( 4 ) M.C. Huysmans 
2. Désaceord des ministres.

La plupart des gouvernements se disloquèrent d'eux-mêmes à la suite d'un désaccord entro los ninistros.

Ie gouvernement tripartite de $M_{0}$. Delacroix démissionne le 25 aout I920 ( I ) parce que, en I'absence du ministre des affalres étrangères, $M$. Hymans, il avait refusé au gouternement français lo droit de transit des munitions destinées à la Pologne en guerre avec la Russie soviétique. M. Hymans démissionra et tout le gouvernement fut entrainé dons la crise parce que les libéraux désiraienti mettre fin à un gouvernement trop tributaire, à leur goût, de la poussée socialisto et flamande. Les socialistes étalent hostiles a toute intervention militairo contre $I^{\prime} U_{0} R_{0} S_{0} S_{0}$, ot les flamands s'on prenalont à la francophilie de $\mathrm{M}$. Hymans ( 2 )。

Le gouverne ment tripartite de M. Carton de wiart se disloqua lo I9 odtobre I92I à la suite d'un conflit entre $M$. Deveze, ministre de la Défense nationale, $\theta^{t} M$. Anseole, g\%1 avait assisté à un meeting socialiate violement antimilitariste à La Louvière.

Le gouvernement catholique-libéral de M. Theunis démissionna le 5 avril I925 parce que les ministres ne pouvalent se mettre d'accord sur l'opportunité dintroduire le vote des femmes aux élections provinciales. Devant l'impasse, le gouvernement s'est décidé à dissoudre les chambres ; Ies élections ourent lieu le 5 avril.

Le gouvernement tripartite de M. Jaspar démissionno le 21 novembre I927 parce que les ministres libéraux s'insurgeaient contre la propagande antimilitariste du parti socialiste qui avait des ministres dans l'équipe ministériello.

Le gouvernement catholique-libéral de $\mathrm{M}_{\circ}$ Jaspar démissionna en mal I93I parce que le premier ministre avait manifesté son accord sur un amendement d'un député catholique flamand tendant à diminuer les crédits prérus jour les fortifications militaires. Les ministres libéraux offrirent imméd Latement leur démission. C'était la troisiame pois en quelques mois ( 3 ). La cause réello de cette crise était le désaccord des ministres sur la question Iinguistique $(4)$.

Le gouve:nenent catholique-libórai de $M_{0}$ Fienkin démissionna 10 I8 octobre I932 parce que les ininistres ne parvenaient pas à se mettre d'accord sur l'opportunité de proośder à des élections gánérales afin de former un gouvernement durable pour affronter la crise économique et les mesuros impopulaires qu'elle appelait. Ie Roi torma un gouvernement de transition, présidé par M. do Broquevilie, pous dissoudro le parlement et orgnnser les élections.

Le gourennemertit dunion rationale de M. Van Acker, le I9 juin I945, et le gouvernement P.S.O. - j. Lbérel de M. Eyskens, le 4 juin I950, prirent fin paroe que les ministres ne pouvatont se mettre d'accord sur l'opportunité du retour du Roi Léopold.

\footnotetext{
IT Le Roi, absert, ne regut ia lettre de demission que le 2 novembre I920.

(2) O.H. Höjer; OF, Cit., F; I09.

(3) Il y out en novembre Ijizo et en janvier I93I, deux crises avortées dont le motif était ia question linguistique ot notarnent le problème de la flamendisation de I universtíe de Gand.

(4) C.H. Höjer, Op. olt: D. IPB.
} 


\section{Déoision des états majors des partis.}

Il est difficile d'établir une nette distinction entre le désac-. cord des ministres et celui des partis. La lialson entre les ministres et leur perti est très étrolte et leurs décisions se confondent la plupart du temps. assez flagrante.

Nous citerons donc ici les cas où l'intervention des partis est

Le gouvernement catholique-lib eral de M. Jaspar, en I929, fut déchiré par la question linguistique. Ies ministres libéraux quittèrent le gouvernement le 25 novembre I929 pour forcer leur parti à accepter la flamandisation de I'université de Gand. La manoeuvre réussit et le Rol refusa la démirnion des ministres libéraux le 4 décombre. Afin d'éviter le sabotage de cette nouvello université, il avait été entendu que les professeurs de cette université ne pourraient donner des cours en Irançais à I'Institut des hautes études de Gand, dont I'unique langue était évidemment le français. Ia fédération bruxelloise du parti libéral désavoua lo ministre libéral de l'Instruction publique, M. Hymans. Il démissionna, et avec lui, tous les ministres libéraux. on jentier I93I. Mais le Roi refusa ces démissions et joua donc un rôlo da frein salutaire à la stabilitë gouvernementale. Les ministres s'inclinèrent apparement, mals les offorts du Roi furent vains; la criso paraissait iném vitable; on mai I93I, usé par la querelle linguistique, le gourernement s'écroula au premier prétexte venu ( I ).

Le gouremement d'union nationale de M. Plerlot, constitué à la I1bération du territolre, démissionna en février I945, les ministres du ravitaillenent, des affaires économiques et de l'agriculture ne parvenont pas à se mettre d'accord sur la politique à suivre. Les ministres socialistes décldèrent de démissionner sur ordre du bureau de leur part1, mais par respect des formes constitutionnelies, attendirent le vote de méfiance du parlement. Les groupes Iibéraux et socialistes annoncèrent on effet qu'ils ne soutiendraient plus le gouvernement. Le premier ministre démissionna sans attendre lo vote officlel " pour que les Belges ne paralssent pas divisés aux yeux de l'étranger et pour faciliter la formation du nouveau gouvernement " (2). La crise était surtout d'ordre personnel. Les socialistes désiraient prendre la direction d'un gouvernement constitué sur les mêmes bases mais dorniné par la forte personnalité de M. Van Ácker.

Le gouvernement homogène de M. Duvieusart, la premier gouvernement catholique homogène depuis I9I4, dut démissionner au mois d'août I950 après I'accession du Prince Baudouin à la régence. Il était coupable, aux yeux de son propre parti, d'avoir dû, devant l'émeute, accepter l'effaccoment du Roi Iéopold.

Le gouvernement P.S.C. homogène de M. Pholien démissionna en décembre I95I sans vote de méflance du parlement, sur injonction discrète, semblet-il, du parti social-chrétien, en raison de l'inaptitude du premier ministre en matière économlque ( 3 ). Le pays devait faire face, on effet, aux conséquences économiques de la guerre de Corée.

\section{Pression d'un groupe parlementaire.}

Le gouvernement de M. Janson, d'union nationale, constitué au mois

(I) Voir Infra, p. 4.

(2) "Le Soir " du B février I945.

( 3 ) Voyez l'appréclation de " La Nation Belge " du I4 arril I954. 
de novembre I937, dut dómissionner six mols après sa constitution, au mois de mal I938 ( I), peroe que les ministres de tendanco conservatrice avaient perdu la confiance, non de la majorité parlementalre ni même celle de leur pert1 pris dans son ensemble, mais bien de leur groupe conservateur. Le motif était tiré de la politique fiscale que le gouvernement se proposait de faire et qui eût certainement trouvé l'appui d'une majorlté parlementaire.

Le gouvernement d'union nationale de M. Spaek se disloqua au mols de févxier I939 à cause de I'incident suivant : un docteur en médecine, du nom de Maertens, condamné à mort par défaut pour avoir collaboré avec les Allemando pendent la première guerre mondiale, puis amnistié, avait été nommé membre de l'Académie flamande de médecine; la nomination fit scandale. Le gouvermement obtint pourtant la confiance de la Chambre par 88 volx contre 86. Ia majorité était une majorité de rechenge, les nationalistes flamands, groupe d'opposition, avaient voté pour le gouvernement, la plupart des 11béraux, contre. Ies ministrés libéraux démissionnérent sous l'influence do leur groupe politique.

Le dernier gouvernement belge avant I'invasion allemande, un cabinot deunion nationale présidé par $M$. Pierlot, ŕisque à con tour de se disloqueì lo 26 arril I940, victime de la querelle Iinguistique ( 2 ). les libó, raux décidèrent de voter contre le budjet de I'Instruction publique, sens voter toutefois contre le gouvernement. Le vote négatif de 20 libéraux entralna pourtant la démission des ministres libéraux, Cette démission so heurta au refus du Roi. Ie IO mai I940, les armées hitlériennes fonçaient à travers la Belgique. Ie rapprochement de ces deux faits souligne l'effrayante fragilité du régime $(3)$.

\section{Causos étrangères aux partis et aux groupos parlementalres.}

Le gouvernement Poullet-Vandervelde, issu des elections du 5 avill I925, après 73 jours d'une laborieuse formation, avait consacré un des plus gros succès électoraux du parti soclaliste en s'appuyant sur une majorité bipartite composée du parti socialiste et d'une importante fraction cathoIlque; l'aile la plus conservatrice de ce parti avait menifesté s-n opposition en votant contre le nouveau gouvernement (I2 voix) ou en s'abstenant ( I3 volx ) ( 4 ). I'opération la plus délicate que devalt réaliser ce gouvernement était la stabilisation de la monnaie. Ia politique qu'il poursuivait dans ce but fut approuvée par le parzoment. Mais la réussite de la réformo monétalre dépendait essentiellement $d$ 'un emprunt anglais. Les négoolations poursuivies à Londres subirent un cuisant échec le Io mars I926 à cause des conditions Impossibles imposées par les créditeurs. Une panique s'empara des milieux financiers belges ot le gouvernement assista, impuiasent, à la fulte des capitaux. Les difflcultés de la Banque nationale devinrent sérieuses. Au mois de mai I926, le franc était tombé à presque la moltié de sa valeur en

TI A.P. Ch., Io-II mai I938, p. I562 - Démission des ministres catholiques conservateurs, le I2 mai, démission du gouvernement entier, le I3 mai I938.

$\left(\begin{array}{l}2 \\ 3\end{array}\right)$ Voir supra, chapitre IV, p. ment ment P.S.C.-P.S.B. de M. Spaak le I8 novembre I948. Le gouvernement démlssionna devant les orires di four contradictolres déposés par las groupes P.S.C. et P.S.B. de la Chombre, Iors tru tatemplation au sujet de le grâce accordéo par le ministre de la Justioo à deux trarase. condamnés à mort pour faits commis sous l'occupation allemande. Le gouvernement très légèrement remanié, se reconstitua immédiatement. Lo ministre de la Justice démissionna avant que la Chambre ne passe au vote. A.P. Ch. I8 novembre I948, p. $27 \%$

(4) A.P. Ch. 23 juin I925 $\theta$ \% jours sulvants. 
litres sterling. Le gouvernement démissionna sans vote de méfiance des chambres, avec une majorité intacte. Il tombiit devant la méflance des miIleux finandiers belges et étrangers ( I ). La cause de son échec était insorite dans les conditions de sa formation : les mêmes milieux qui causérent sa perte s'étaient employés, en vain il est vral, à empêcher sa nalssanoo.

Le gouvernement présidé par M. Theunis, formé de catholiques, de Ilbéraux et de personnalités extraparlementaires venues des milieux finanolers, dut également démissionner au mois de mars I935, pour le motif essentiel que la situation monétaire était devenue intenable et qu'une dévaluation du frane paraissait inévitable ( 2 ). La situation parut assez grave pour justifier la formation d'un gouvernement d'union nationale, c'est-à-dire d'une coalition des trois plus grands partis, catholique, libéral et socialiste. Présidé par M. Van Zeeland, ce gouvernement avait pour ţ̂cho essentielle de réallser la dévaluation du franc. Le gouvernement de $M$. Theunis ne chercha pas s'il y avait au Parlement une majorité suffisante pour appuyer l'opération que M. Van Zeeland allait pourtant accomplir.

Le 25 octobre I937 le gouvernement démissionna devant les attaques personnelles visant son chef, $M_{c}$ Van Zeeland, Les adversaires politiques de ce dernier l'accusaient de payer de larges gratifications à ses attachés de oabinet à I'aide d'un fonds secret de la Banque nationalo. I'affaire do Ia " cagnotte " entraina la démission collective du gouvernement, malgré I'appui parlementaire dont il jouissait ( 3 ). Elle avait été exploitée à fond par les rexistes, les nationalistes flamands et un hebdomadaire d'extrôme-droite, "Cassandre $"$. A la suite de nouvelles découvertes relatives à la Banque nationale, dont $M$. Van Zeeland avait été vice-gouverneur, une instruction judiciaire fut ouverte, qui ne visait pas le premier ministre malo qui mit de l'hulie sur le fou. Le premier ministre préféra démissionner $(4)$.

\section{Conclusions.}

Les gouvernements sont paralysés par la règle de I'unanimité. Cette règle s'étend parfois du conseil des ministres jusqu'aux groupes parlementaires de la majorité et aux partis dont ils sont la projection électorale.

Si cette unanimité est rompue pour une raison quelconque, le gouvernement s'écroule.

Si les gouvernoments subissent la pression des groupes parlementaires et des partis, nous avons vu que les gourernements le leur rendent bien.

La démission s'est révélée souvent conme un moyen de pression efficace pour obliger un groupe parlementaire ou un parti a se rallier même de mauvals gré, à la politique gouvernementale,

Le régime parlementaire est le régjme de la confusion entre gouverm nement, parlement et partis. La démocratie gagnerait pourtant à promouvoir I'Indépendance réciproqúe de ces trois éléments.

Quant à la responsabillté ministếrielle, principe constitutionnel

\footnotetext{
(I) Volr Histolre economique de la Belgique, F, Baudhuin, p. I60.

(2) sur la crise ministérielle belge, vojez C.H. Eöjer op. cit., p。 235.

(3) Annales parlementaires Chambre 7 août I937; $p$.

(4) Voir C.H. Höjer, op. cit. p, $265-267$.
} 
sur lequel tout le régime est basé, elle s'exerce égelement dans une grande confusion.

I'histoire des crises ministérielles soullgne bien le rôle réel du parlement. Les deux assembIées ont pour rôle essentiel de maintenir un gouvernement au pouroir et d'entériner sa politique, sa législation et jusqu'à ses moindres actes, ou bien de rejeter un gouvernement dans le néant politique, à charge d'approuvcr Ia formation et le programme du successeur.

Quant ì vouloir trancher un point prócis et celui-là soulement, il n'en est pas question. La responsabilité ministérielle porte sur tant de choses à la fols et se situe nécessairement sur un plan si général qu'elle on devient complètement diffuse, pour ne pas dire inexistante.

\section{REIATIVITE DE L' INSTABILITE MINISTTERIELIE。}

Nous avons dénombré 22 crises ministérielles en Belgique. Nous n'arons pas tenu compte des remaniements assez fréquents qui ne portaient que sur la personne de quelques ministres, encore que ces changements n alent pas toujours été dépourvis de signification politique. En en tenant compte, los orises auraient atteint la trentaine ( $I$ )。

Cette instabilité n'est pas absolue : à chaque crise gouvernementale, le gouvernement ne meurt jeinals tout entier. Ies ministres démissionnaires constituent souvent le tiers, la moitié ou même plus, de la nouvelle équipe ( 2$)$.

D'autre part, certains ministres restent assez longtemps à la tôte du même département, on faisent partie de gouvernements successifs.

M. Hymans, par exemple, fut ministre des affaires étrangères pendant IO ans, de I9I8 à I935, mais en p'jojos successives, évaluées approximativement $a \mathrm{a} 2, I, 6, I$ ans, avec des interruptions allant de quelques mols à 2 ou 3 ans. M. P.H. Spaak, qui semble battre le reccord de la stabilité ministórlelle personnelie, a dirigé les affaires étrangères du pays, du mo1s de juin I936 au mois de juin I949 (' 3 ), et du mois d'avril I954 au mols de mars I957. M. Devèze fut ministre de la défense nationale pendant trois périodes successives : 3 , 4 et $I$ an; $M_{0}$ de Broqueville occupa lo môme poste pendent 5 ans sans interruption, 10 général Denis pendant 4 ans, le oolonel Defraiteur pendant plus de 3 ans. MM. Wauters, Moyersoen, Hejman, Delattre, Troclet, ont été ministres du travail et de la prévoyance sociale pour des périodes allant de 3 à 5 ans. Ce sont là des durées minima en-dessous desquelles un régime qui fonotionne blen ne cevrait pas descendre. Mais ces trois départements sont priviléglés. Ies autres passenti dune main à l'autre avec une rapidité déconcertente, surtout après I934. En 6 ans, 11 $\mathrm{y}$ out 9 ministres de I'intérieur, 9 à íinstruction publique, 9 à la justLeo, 7 aux finances, tte... I"aprés-guerre de I944 à I958 a connu 8 minis* tres de l'Intérieur, 7 ministres des finances, Ia ministres de la justice, eta...

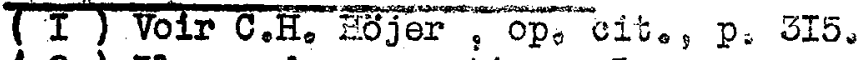

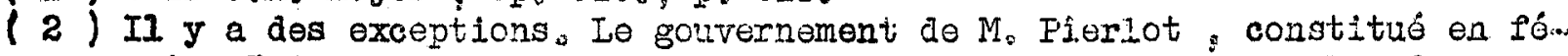
vrier I939, ne comptait plus que deux anciens ministros celui de $M_{0}$ Pholien, au mois d"eot' I950, $\mathrm{n}^{\prime}$ en comptait que deux égalemento

(3) arec seulement une interruption te quelques mois, du mois de janvier au mols de septembre 1939.
} 
Le personnel politique dirigeant varie peu. Il y a quelques hommes politiques qui mènent le jeu à titre ossentiel pendant de longues annóes et qui occupent des charges minlstérielies d'une manière fort constante.

On peut citer les noms de MM. Jaspar, Hymans, de Broqueville, Poullet, Devèze, Janson, Theunis, Houtart, Heyman, Vandervelde, Anseele, Wauters, Iippens pour la période qui va de I9I8 à I935. Ajoutons-y pour I'avant et I'après-guerro, MM. Van Zeeland, Plerlot, Spaak, De Schrijver, De Vleeschauwer, Bovesse, Jaspar, Merlot, De Man, Delattre, Wauters, Van Acker, Hyskens, Huysmans, Troclet, Segers; Behogne, Van Glabbeke, Buisseret, Rey, Vermeylen. permanence.

Ces hormes dirigent réellement la politique belge avec assez de

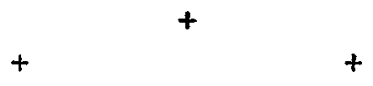

D'autre part, une plus grande stabilité enoore rôgne en ce qui conoerne les alliances politiques des gouvernements successifs. Ce fait n'a rien de surprenant. Aucun part1, entre novembre I9I8 et juin I950, n'obtint la majorité absolue. Les Partis furent donc contraints de se cooliser. Le parti catholique gouverna sans interruption jusqu'en I945 et s'allie assez souvent avec son ancien adversaire de la période bourgeolse, le perti libéral, contre le parti socialiste ( I ). Cette collaboration a donné les gouvernements les plus stables (2) que la Belgique ait connus de I9I8 à I958, à I'exception du gouvernement de guerre exilé à Londres et du gouvernement socialiste-libéral de M. Van Acker ( I954I958).

Les páriodes les plus critiques ont vu naitre des gouvernements d'union nationale, (avec les trois partis, catholique, libéral et socialisto / constitués soit à cause des ãifficultés à résoudre, soit pour faire face à l'opposition d'extrême-droite des rexistes et des nationalistes flamands; ils furent instables mais assez novateurs et énergiques ( 3 ).

Très rarement le parti catholique s'unit au parti socialiste seul; cette expérience compte deux úchecs $(4)$ et une réussite ( 5 ), soit en

TI Gouvernement Theunis du I6.I2.I92I au I3.5.I925; du 22.II.I927 au 20.II.I934, deux gouvernements Jaspar , gouvernement Renkin, gouvornement de Broqueville, gouvernement Theunis ; du I8.4.I939 au 3,9.I939: gouvernement Pierlot; de août I949 à juin I950, gouvernement Eyskens: o'est-à-dire I3 ans de collaboration.

(2) Ie gouvernement Theunis du I6.I2.I92I au I3.5.I925; le gouvernement Jaspar du 22.II.I927 au 6.6.I93I.

( 3 ) du 2I.II.I9I8 au 20.II.I920; deux gouvernements Delacroix, gouvernem ment Carton de wiart; du $20,5, I 926$ au $22 . I I$.I927, gouvernement Jaspar; du 25.3.I935 au 22.2.I939, deux gouvemements Van Zeeland, gouvernement Janson, gouvernement spaak ; du 3.9.1939 au mois de juillet I945, goum vernoment Pierlot, gouvernement, de Londres, gouvernement Pierlot après la libération, gouvernement $\operatorname{Van} A$ deker, soit en tout plus de II ans de collaboration, $y$ compris 5 ans de guerre.

(4) Poullet-Vandervelde du I7.6.I925 au 20.5.I926; Pierlot du 22.2.I939 au I8.4.I939.

( 5 ) P.- H. Spaak du mois de mars I947 au mois de juin I949. 
I8.

tout presque trois ans et demi de collaboration sur une póriode de quarante ans.

Signalons enfin les gouvernements des gauches ( libéraux, soclalistes, communistes ) du mols de juillet I945 au mols de mars I947 ( I ), do nt la formation ne s'explique que par l'abstention volontaire des sociaux-chrétiens partisans du Ro1 Léopold. Ajoutons-y l'alliance Ifbéralo-socialiste I954-I958, réplique aux gouvernements P.S.C. homogènes I950-I954.

I'alliance catholique-libérale et le tripartisme d'union nationale dominent dono la vie politique belge pendant la pérlode d'entre deux guerres; après I944, au contraire, I'union nationale (quadripartite) ne dura pas six mois, et l'alliance libérale-catholique moins d'un an.

Quol qu'il on soit, les alliances politlques on Belgique offrent des veriantes relativement peu nombreuses. II n'y a pratiquement que trois partis politiques qui se partagent lo pouvoir. Une grande stabilité règne dono dans les traits fondamentaux de la politique. Cette stabilité est celle même de l'opinion publique qui varie peu. Les crises gouvernementales issues des querelles intestines des coalitions ne traduisent jamais un revirement complet de l'orlentation politique. Au fond, on n'aporçolt pas bien leur utilité. D'une élection à l'autre, les données du problène ne changent presque jamais. I'élection donne une tendance qui peut inspirer la politique jusqu'à l'élection suivante. Les changements de gouvernement dans l'intervalie - remantements utiles mis à port - ne correspondent à auoune néoessité profonde. Ie plupart du temps, les crises gouvernementales ne servent pas à résoudre réellement les difficultés qui les ont provoquées. Renverser le gourernement $n^{\prime}$ est pas tout, il faut encore pouvoir y substituer une autre équlpe qui fera un toute autre politique. Sans cela, la crise est vaino.

La dislocation des équipes gouvernementales ne servant la plupart du temps a rien, ne faudrait-1l pas s'en passer et chercher un autre moyen de falro trancher les questions au sujet desquelles les ministres ne parviennent pas à se mettre d'accord ?

Les orises ministérielles, si irritantes pour l'opinion publique, -1 préjudiclables au crédit du rógime et par conséquent à la démocratie politique, ne révèlent-elles pas que certaines règles du jeu parlementairo ne sont pas adaptées à la réalité politique du pays ? Nous obéissons à des règles de jeu en faveur en Grande-Bretagno, mals nous n'avons pas les memes cartes que los Eritanniques. Tout le jeu en est faussć. Nous ne devons pas falre come les mauvais joueurs et dire arec humeur que les cartes sont mauvaises. Nous devons faire preuve d'imagination et inventer des règles qul nous sont propres, on tenont compte des atouts que nous arons.

(I) Gouvernement Van Acker qui comptait des catholiques indépendents (U.D.B) sans représentation parlementaire et qui devaient échouer totalement aux élections de février I946; un second gouvernement Van Acker, après un essal inIruotueux de gouvernement soclalis te homogène de P.H.Spaak; un gouvernement Huysmans. 